\title{
Measurement of cerebrovascular reserve by multimodal imaging for cerebral arterial occlusion or stenosis patients: protocol of a prospective, randomized, controlled clinical study
}

\author{
Zhi-peng Xiao ${ }^{\dagger}$, ke Jin ${ }^{\dagger}$, Jie-qing Wan, Yong Lin, Yao-hua Pan, Yi-chao Jin and Xiao-hua Zhang ${ }^{*}$ (1)
}

\begin{abstract}
Background: Cerebrovascular reactivity (CVR) is the change in cerebral blood flow in response to a vaso-active stimulus, and may assist the treatment strategy of ischemic stroke. However, previous studies reported that a therapeutic strategy for stroke mainly depends on the degree of vascular stenosis with steady-state vascular parameters (e.g., cerebral blood flow and CVR). Hence, measurement of CVR by multimodal imaging techniques may improve the treatment of ischemic stroke.
\end{abstract}

Methods/design: This is a prospective, randomized, controlled clinical trial that aimed to examine the capability of multimodal imaging techniques for the evaluation of CVR to improve treatment of patients with ischemic stroke. A total of 66 eligible patients will be recruited from Renji Hospital, Shanghai Jiaotong University School of Medicine. The patients will be categorized based on CVR into two subgroups as follows: CVR $>10 \%$ group and CVR $<10 \%$ group. The patients will be randomly assigned to medical management, percutaneous transluminal angioplasty and stenting, and intracranial and extra-cranial bypass groups in a 1:1:1 ratio. The primary endpoint is all adverse events and ipsilateral stroke recurrence at 6,12 , and 24 months after management. The secondary outcomes include the CVR, the National Institute of Health stroke scale and the Modified Rankin Scale at 6, 12, and 24 months.

Discussion: Measurement of cerebrovascular reserve by multimodal image is recommended by most recent studies to guide the treatment of ischemic stroke, and thus its efficacy and evaluation accuracy need to be established in randomized controlled settings. This prospective, parallel, randomized, controlled registry study, together with other ongoing studies, should present more evidence for optimal individualized accurate treatment of ischemic stroke.

Trial registration: Chinese Clinical Trial Registry, ID: ChiCTR-IOR-16009635; Registered on 16 October 2016. All items are from the World Health Organization Trial Registration Data Set and registration in the Chinese Clinical Trial Registry: ChiCTR-IOR-16009635.

Keywords: Stroke, Cerebrovascular reactivity, Cerebral blood flow, Multimodal image, Recurrence

\footnotetext{
* Correspondence: zxh1969@aliyun.com

†Zhi-peng Xiao and ke Jin contributed equally to this work.

Department of Neurosurgery, Renji Hospital, School of Medicine of Shanghai

JiaoTong University, Shanghai 200127, People's Republic of China
}

(c) The Author(s). 2020 Open Access This article is distributed under the terms of the Creative Commons Attribution 4.0 International License (http://creativecommons.org/licenses/by/4.0/) which permits unrestricted use, distribution, and reproduction in any medium, provided you give appropriate credit to the original author(s) and the source, provide a link to the Creative Commons license, and indicate if changes were made. The Creative Commons Public Domain Dedication waiver (http://creativecommons.org/publicdomain/zero/1.0/) applies to the data made available in this article, unless otherwise stated. 


\section{Introduction}

Stroke is the second most common cause of death and the major cause of disability worldwide after ischemic heart disease, especially in developing countries [1]. Ischemic stroke occurs when a blood vessel supplying blood to a part of the brain is obstructed, and it accounts for about $87 \%$ of all strokes. A remarkable risk of recurrent ischemic stroke was reported in patients with symptomatic, major cerebral arterial occlusion or stenosis [2]. Therefore, an effective therapeutic approach for intracranial arterial stenosis is urgently required.

The treatment of ischemic stroke has been investigated by a number of high-quality trials: The Japanese extracranialintracranial bypass (EC-IC) bypass trial (JET2) study revealed that compared with the medical arm of the Japanese EC-IC bypass trial (JET) study including patients with cerebral blood flow $(\mathrm{CBF})<80 \%$ and cerebrovascular reactivity $(\mathrm{CVR})<10 \%$ as a historical control, the incidence of ipsilateral stroke recurrence was significantly lower in the JET2 study, demonstrating that EC-IC bypass surgery is unlikely to benefit patients with $\mathrm{CBF}>80 \%$ or CVR $>10 \%$ [3]. Another recent trial involving aggressive medical treatment with or without stenting in high-risk patients with intracranial arterial stenosis (SAMMPRIS), demonstrated the use of aggressive medical management rather than percutaneous transluminal angioplasty and stenting (PTAS) with the Wingspan system in high-risk patients with atherosclerotic intracranial arterial stenosis [4]. AL Hasan conducted a trial for treating ischemic stroke, and showed a $14.7 \%$ risk of stroke or death in the stenting group versus $5.8 \%$ in the medical group at 30 days, and $23 \%$ in the stenting group versus $15 \%$ in the medical group at a median follow-up of 32.4 months. However, the treatment strategy of intracranial arterial stenosis or occlusion mainly depends on the degree of vascular stenosis, with or without consideration of hemodynamic factors at the distal end-to-side anastomosis of a bypass graft and CVR factors, or steady-state vascular parameters, such as CBF and cerebral blood volume (CBV). Hence, we present a study protocol for the measurement of CVR using multimodal imaging data for cerebral arterial occlusion or stenosis patients.

The CVR is the ability of cerebral vessels to dilate or constrict in response to challenges or maneuvers $[5,6]$. In addition, CVR is thought to be an important index of the brain's vascular health, and provides vascular-reserve information that is complementary to steady-state vascular parameters, including CBF and CBV $[7,8]$. There have been two main approaches to measuring CVR. One approach attempts direct CBF measurements of the brain tissue with flow-sensitive imaging techniques such as positron-emission tomography (PET), nuclear medicine (NM) techniques, computed tomography $(\mathrm{CT})$ perfusion, or magnetic resonance imaging (MRI) perfusion before and after a vasodilatory stimulus. The second approach involves transcranial Doppler (TCD) measurement of flow velocities (typically in the middle cerebral artery (MCA)) distal to a lesion both before and after a vasodilatory stimulus, with the increase flow velocity considered a surrogate for CVR [9-12]. We intend to precisely evaluate the change of CVR before and after surgical or medical treatments by multimodal image including MRI, CT, and single-photon-emission computed tomography (SPECT), so that we can make strategies for individualized accurate diagnosis and treatment for the ischemic stroke $[3,13]$.

Due to the lack of effective therapeutic approaches for intracranial arterial stenosis or occlusion, the present trial was registered at the Chinese Clinical Trial Registry database, and approved by the Center for Reproductive Medicine at Renji Hospital (Shanghai, China). The present trial was designed to determine whether multimodal imaging data can effectively enhance the treatment strategy for adult patients with intracranial arterial stenosis or occlusion.

\section{Methods/design Study design}

This prospective, randomized, controlled clinical trial aimed to examine the efficacy of multimodal image data based on CVR to treat ischemic stroke. A total of 66 patients, who met the inclusion criteria, were admitted to Center for Reproductive Medicine at Renji Hospital, Shanghai Jiaotong University School of Medicine (Shanghai, China). The eligible patients were categorized based on CVR into two groups as follows: CVR $>10 \%$ group and CVR $<10 \%$ group. In addition, these two groups were randomly assigned to the groups of medical management, single angioplasty, PTAS, and IC-EC bypass in a 1:1:1 ratio. Fig. 1 shows the study flowchart of our trials.

\section{Inclusion and exclusion criteria}

The eligible patients were identified if they met the following criteria: (1) clinical requirements: (a) men and women aged between 18 and 70 years, (b) independency in the activities of daily living (modified Rankin scale score of 0-2) on admission or after resuscitation; (2) radiological requirements: (a) occlusion or severe stenosis in the main trunk of the MCA or the supraclinoid segment of the internal carotid artery, (b) CT and MRI: no large infarction and no contrast enhancement in the infarcted area; and (3) signing the written informed consent form.

Exclusion criteria were as follow: (1) no independency in the activities of daily living (modified Rankin scale score of 3-5); (2) occlusive lesions of the cerebral arteries due to diseases other than atherosclerosis; (3) malignant tumors or multi-organ dysfunction involving the heart, liver, kidney, or lung; (4) myocardial infarction within the past 6 months; (5) uncontrolled diabetes 


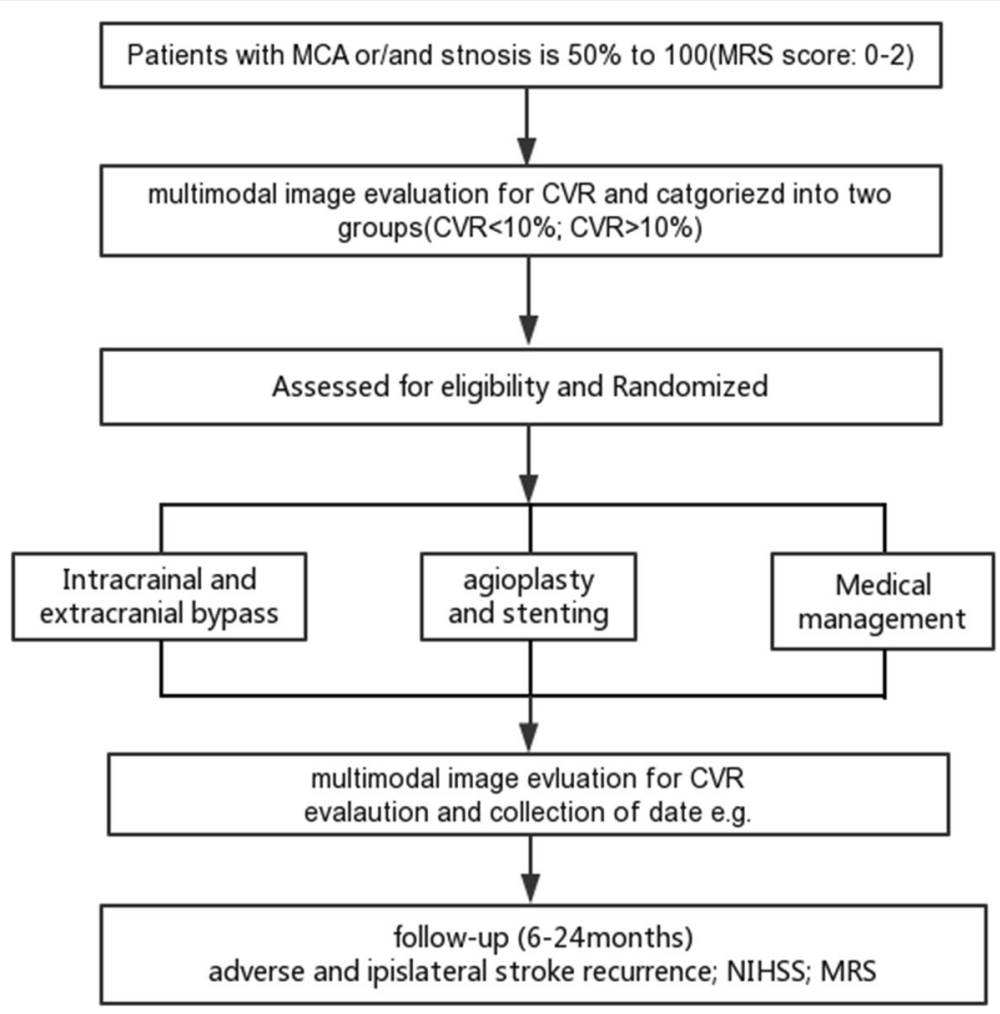

Fig. 1 Study flowchart of our trial

showing a serum fasting blood glucose level of $>300$ $\mathrm{mg} / \mathrm{dL}$, or requiring insulin; (6) hypertension with a diastolic blood pressure of $>110 \mathrm{mmHg} ; 7$; (7) artery-toartery embolism; and (8) cardioembolism.

\section{Ethical approval and consent to participate}

The study protocol was approved by the Ethics Committee of Renji Hospital. Furthermore, the present trial was registered at the Chinese Clinical Trial Registry (Registration No. ChiCTR-IOR-16009635). The trial was performed in accordance with the Declaration of Helsinki. All the participants signed the written informed consent form prior to start of study. The principal investigator explained the content of the research plan with the patient; including whether they agree to use of their data and asked for permission for the research team to share their relevant data with people from the universities taking part in the research or from the regulatory authorities. The principal investigator will obtain informed consent or assent from potential trial participants or the patient's legal representative [14].

\section{Randomization and allocation concealment}

In this trial, randomization sequence was generated by an independent institution that was not involved in the determination of eligibility.

\section{Treatment protocol}

The eligible patients underwent multimodal imagine to measure CVR. Regional CBF was quantitatively measured more than 3 weeks after the last ischemic attacks using computed tomography perfusion (CTP) or SPECT (123IIMP). A study of a small number of patients with chronic arterial stenosis compared ASL perfusion with ACZ challenge with iodine $123 \mathrm{~N}$-isopropyl-piodoamphetamine (123I-IMP) SPECT, and the fixed concentration of $\mathrm{CO}_{2}$ was provided by a gas-delivery system using a pressure transmitter to control the gas blender with prospective gastargeting algorithms. Subjects underwent SPECT/CTP scanning, and were asked to breathe normally for $10 \mathrm{~min}$. The region of interest (ROI) was designated manually in the cerebral cortex in the territory of the ipsilateral MCA at the level of the anterior horn of the lateral ventricle. ROIs were also placed in the bilateral cerebellar hemispheres and in the contralateral MCA territory as reference. Regional $\mathrm{CBF}$ was expressed as relative values (\%) to normal control values of each institute obtained from volunteers free of cerebrovascular disease. CVR was calculated as follows:

$$
\text { CVR }(\%)=\left[\left(\mathrm{CO}_{2} \text { challenge } C B F-\text { rest } C B F\right) / \text { rest } C B F\right] \times 100 .
$$

All treatment procedures and specific operating protocols for the management of ischemic stroke in our trial were standardized based on current guidelines. 
Surgical intervention was microsurgical end-to-side anastomosis of a superficial temporal artery branch to a cortical branch of the MCA. If the superficial temporal branch was felt to be inappropriate, the occipital artery could be used. For participants in the surgical group, preoperative and postoperative antithrombotic treatments were carried out by a neurosurgeon. Patients in the PTAS group received stenting when "alarm" symptoms were relieved after MT. Participants in the nonsurgical group continued to receive the antithrombotic treatment preferred by their physicians. Targets for controlling risk factor were $130 / 85 \mathrm{mmHg}$ for blood pressure, $100 \mathrm{mg} / \mathrm{dL}$ for low-density lipoprotein, 150 $\mathrm{mg} / \mathrm{dL}$ for triglycerides, and 7\% for hemoglobin A1C.

\section{Study endpoints}

Each patient was followed-up for 2 years by a neurologist and a neurosurgeon in each participating institute. Primary and secondary endpoints were defined as all adverse events and ipsilateral stroke recurrence at 6,12 , and 24 months after management, respectively. Neurological findings, intracranial CT/MRI, and CBF/CVR measurements were examined and reported at the time of enrollment and at 6 months, 1 year, and 2 years after enrollment. Evaluation of cognitive function and angiography were carried out at the time of enrollment and 2 years after enrollment. The functional outcomes were measured using CVR, the National Institute of Health stroke scale (NIHSS), and the Modified Rankin Scale (MRS) at 6, 12, and 24 months after the management.

\section{Data collection}

The baseline data were collected including the following variables: hypertension, smoking status and whether diabetic, clinical presentation (i.e., initial ischemic stroke, on admission and before treatment); neurological functions (NIHSS and MRS), vascular stenosis, timing of management, treatment procedure, neurological conditions within $72 \mathrm{~h}$ after treatment, complications during hospitalization, follow-up, and presumed reasons of death. There is no storage of biological specimens for genetic or molecular analysis in the current trial and for future use in ancillary studies.

\section{Follow-up}

In this trial, CTP and SPECT were followed-up 6 months after treatment. All the patients were followed-up after management by a neurosurgeon using a telephone interview or an in-person interview. The neurosurgeon was trained before the registry and was not involved in the treatment of ischemic-stroke patients. In outcomes after 6,12 , and 24 months, a MRS of 0-2 denoted a satisfactory outcome, and a score of 3-6 denoted a poor outcome.
Data verification was undertaken in $20 \%$ of all cases to assess the accuracy of data collection. The monthly audit, check of data quality, and statistical analysis were conducted by a third party who was in charge of notifying the principal investigator and Institutional Review Board of Renji Hospital about any issues that had arisen. Any serious adverse events were reported to the Institutional Review Board of Renji Hospital. Recommendations were forwarded to the principal investigators for reviewing risks and benefits. The Institutional Review Board had access to the interim results and made the final decision to terminate the trial.

\section{Sample size and data analysis}

The number of patients included in the registry was equal to 60, and this trial involved 66 eligible ischemicstroke patients, in which about $10 \%$ of patients were lost to follow-up. Data were presented as mean \pm standard deviation (SD) for continuous variables, and as frequency for categorical variables. Significances between variables were analyzed using the chi-square test. Associations between clinical variables and outcomes were analyzed, and predictors of long-term outcome were identified using univariate and multivariate regression analyses. The difference was expressed as an odds ratio (OR, with 95\% confidence interval $(\mathrm{CI})$ ), and $P<0.05$ was considered statistically significant.

\section{Discussion}

Previous studies have shown that medical management, PTAS, and IC-EC bypass can be applied to ischemicstroke patients; however, which treatment method is more beneficial has remained elusive $[4,15]$. The present trial was designed to indicate whether measurement of CVR using multimodal imaging is helpful to improve the treatment strategy for adult patients with intracranial arterial stenosis or occlusion.

Increased risk of stroke was found to be associated with hemodynamic failure, which can be assessed with measurement of CBF using $\left({ }^{15} \mathrm{O}-\right) \mathrm{H}_{2} \mathrm{O}$ PET [16]. This gold-standard technique, however, has not been presented for routine clinical imaging. Standardized blood oxygen-level-dependent functional $\mathrm{MRI}+\mathrm{CO}_{2}$ is a noninvasive and potentially widely applicable method to assess whole-brain quantitative CVR. In addition, SPECT/ CTP combined with $\mathrm{CO}_{2}$ challenge enables scholars to measure $\mathrm{CBF}$ and $\mathrm{CVR}$, representing the degree of hemodynamic failure $[17,18]$.

It has been previously demonstrated that there is an association between CVR impairment and risk of stroke conserved across testing modality (TCD or nuclear medicine (NM) technique) as well as the nature of the vasodilatory stimulus (acetazolamide or variation in inspired $\mathrm{CO}_{2}$ levels). TCD is relatively inexpensive and 
widely available, while it does not provide additional information about brain parenchyma and is technically impossible in some cases due to lack of acoustic windows. In the present study, we accurately evaluated CVR for ischemic-stroke patients by multimodal imaging methods (MRI, CT, or SPECT), and explored the recurrence of ischemic stroke after treatment; thus, we can develop a new method for accurate diagnosis and treatment of ischemic stroke $[9,19,20]$. Our approach possesses a number of novel features compared with other relevant trials. Firstly, CVR in the ischemic-stroke patients was evaluated by multimodal imagine methods because those are widely applicable to assess whole-brain quantitative CVR. Some researchers have reported that SPECT is more sensitive than PET in the evaluation of CBF and cerebral perfusion, while its spatial resolution is negligible [21, 22]. The above-mentioned findings demonstrate that multimodal imaging techniques can accurately reflect changes in CVR. Secondly, the current study categorized the eligible patients into the groups of medical management, PTAS, and IC-EC bypass based on and the rates of CVR.

Finally, we recorded all the data related to the changes of CVR before and after treatment by multimodal imaging. The adverse events during the follow-up period were also taken and divided into ipsilateral stroke recurrence and all adverse events. We consequently found that such data could be help to analyze the effects of relevant confounding factors.

Our trial also indicated whether CVR can effectively and safely help improve the outcome in patients with intracranial arterial stenosis or occlusion. Regarding the major challenges in performing a clinical trial on ischemic stroke, the necessity for huge amounts of data is inevitable.

\section{Trial status}

This protocol is the first version 1 , which approved on 12 May 2019. The trial was started on 6 June 2018. We hope to achieve our research objectives by September 2020 .

\section{Abbreviations \\ CBV: Cerebral blood volume; CTP: Computed tomography perfusion; CVR: Cerebrovascular reserve; EC-IC: Extracranial-intracranial bypass; MCA: Middle cerebral artery; MRI: Magnetic resonance imaging; PET: Positron-emission tomography; PTAS: Percutaneous transluminal angioplasty and stenting; SPECT: Single-photon-emission computed tomography}

\section{Acknowledgements}

The authors express their gratitude to all members of the Clinical Research Center of Renji Hospital for their significant contribution in this study.

\section{Authors' contributions}

XZP, JK, LY, WJQ, PYH, JYC, and ZXH designed the study. XZP and JK drafted the manuscript. JYC and $Z X H$ revised the manuscript. All authors agreed to publish this trial. The data analyzed during the current study are available from the corresponding author on reasonable request. All authors read and approved the final manuscript.

\section{Funding}

This study was funded by Shanghai Shen Kang Hospital Development Center Research Program (Grant Nos. 16CR2048B and 16CR2045B).

\section{Availability of data and materials}

Not applicable

\section{Ethics approval and consent to participate}

The study protocol was approved by the Ethics Committee of Renji Hospital (2016-143 K).

There is no anticipated harm or compensation for trial participation and informed consent will be obtained from all patients enrolled in the study. All the patient data will be uploaded to Electronic Data Capture (China Clinical Trial Database); and submitted to published articles, or presented at a lecture at the Neurosurgery Conference.

\section{Competing interests}

The authors declare that they have no competing interests.

Received: 29 July 2019 Accepted: 6 December 2019

Published online: 08 January 2020

\section{References}

1. Pandian JD, et al. Prevention of stroke: a global perspective. Lancet. 2018; 392(10154):1269-78.

2. Donnan GA, et al. Stroke. Lancet. 2008:371(9624):1612-23.

3. Kataoka H, et al. Results of prospective cohort study on symptomatic cerebrovascular occlusive disease showing mild hemodynamic compromise [Japanese Extracranial-Intracranial Bypass Trial (JET)-2 Study]. Neurol Med Chir (Tokyo). 2015;55(6):460-8.

4. Derdeyn CP, et al. Aggressive medical treatment with or without stenting in high-risk patients with intracranial artery stenosis (SAMMPRIS): the final results of a randomised trial. Lancet. 2014;383(9914):333-41.

5. Liu P, De Vis JB, Lu H. Cerebrovascular reactivity (CVR) MRI with $\mathrm{CO}_{2}$ challenge: a technical review. Neuroimage. 2019;187:104-15.

6. Gupta A, et al. Cerebrovascular reserve and stroke risk in patients with carotid stenosis or occlusion: a systematic review and meta-analysis. Stroke. 2012;43(11):2884-91.

7. Smeeing DP, et al. Arterial spin labeling and blood oxygen level-dependent MRI cerebrovascular reactivity in cerebrovascular disease: a systematic review and meta-analysis. Cerebrovasc Dis. 2016;42(3-4):288-307.

8. Chen DW, et al. Assessment of the cerebral hemodynamic benefits of carotid artery stenting for patients with preoperative hemodynamic impairment using cerebral Single Photon Emission Computed Tomography (SPECT) and carbon dioxide inhalation. Med Sci Monit. 2018;24:5398-404.

9. Boudiaf $\mathrm{N}$, et al. BOLD fMRI of cerebrovascular reactivity in the middle cerebral artery territory: a 100 volunteers' study. J Neuroradiol. 2015;42(6):338-44.

10. Choksi $\mathrm{V}$, et al. Transient neurologic deficit after acetazolamide challenge for computed tomography perfusion imaging. J Comput Assist Tomogr. 2005; 29(2):278-80.

11. De Bortoli M, et al. Cerebral vasoreactivity: concordance of breath holding test and acetazolamide injection in current practice: 20 cases of asymptomatic carotid artery stenosis. J Med Vasc. 2017;42(5):272-81.

12. Seo HJ, et al. Hemodynamic significance of internal carotid or middle cerebral artery stenosis detected on magnetic resonance angiography. Yonsei Med J. 2015;56(6):1686-93.

13. Thomas BP, et al. Physiologic underpinnings of negative BOLD cerebrovascular reactivity in brain ventricles. Neuroimage. 2013;83:505-12.

14. World Medical Association. World Medical Association Declaration of Helsinki: ethical principles for medical research involving human subjects. JAMA. 2013;310(20):2191-4.

15. Al HM, Murugan R. Stenting versus aggressive medical therapy for intracranial arterial stenosis: more harm than good. Crit Care. 2012;16(3):310.

16. Gruner JM, et al. Brain perfusion CT compared with15O-H2O-PET in healthy subjects. EJNMMI Res. 2011;1(1):28.

17. Muscas $\mathrm{G}$, et al. Feasibility and safety of intraoperative BOLD functional MRI cerebrovascular reactivity to evaluate extracranial-to-intracranial bypass efficacy. Neurosurg Focus. 2019;46(2):E7.

18. Taneja $\mathrm{K}$, et al. Evaluation of cerebrovascular reserve in patients with cerebrovascular diseases using resting-state MRI: a feasibility study. Magn Reson Imaging. 2019;59:46-52. 
19. Sebok M, et al. BOLD cerebrovascular reactivity as a novel marker for crossed cerebellar diaschisis. Neurology. 2018;91(14):e1328-37.

20. Fierstra J, et al. Staging hemodynamic failure with blood oxygen-leveldependent functional magnetic resonance imaging cerebrovascular reactivity: a comparison versus gold standard ((15)O-)H2O-positron emission tomography. Stroke. 2018;49(3):621-9.

21. Choi JW, et al. Neural correlates of motor recovery measured by SPECT at six months after basal ganglia stroke. Ann Rehabil Med. 2017;41(6):905-14.

22. Eicker SO, et al. A comparative study of perfusion CT and 99m Tc-HMPAO SPECT measurement to assess cerebrovascular reserve capacity in patients with internal carotid artery occlusion. Eur J Med Res. 2011;16(11):484-90.

\section{Publisher's Note}

Springer Nature remains neutral with regard to jurisdictional claims in published maps and institutional affiliations.

Ready to submit your research? Choose BMC and benefit from:

- fast, convenient online submission

- thorough peer review by experienced researchers in your field

- rapid publication on acceptance

- support for research data, including large and complex data types

- gold Open Access which fosters wider collaboration and increased citations

- maximum visibility for your research: over $100 \mathrm{M}$ website views per year

At BMC, research is always in progress.

Learn more biomedcentral.com/submissions 